\title{
Hepatosplenic Bartonellosis in an Immunocompetent Teenager: An Atypical Presentation of Cat-Scratch Disease
}

\author{
Rohit Sharma ${ }^{1}$, Abdullah Mohammad Arshad ${ }^{1}$, Sundus Sardar ${ }^{1}$, Abdulaziz Zafar ${ }^{1}$ \\ 1. Internal Medicine, Hamad Medical Corporation, Doha, QAT
}

Corresponding author: Rohit Sharma, rohitsharma8692@gmail.com

\begin{abstract}
Infection with Bartonella henselae, a gram-negative coccobacillus, most frequently presents as cat-scratch disease (CSD) and often accompanies a recent history of cat bite or scratch. As compared to adults, teenagers and children or immunocompromised patients are predominantly affected by CSD. In immunocompetent individuals, CSD is typically a self-limiting clinical syndrome with complete resolution of febrile illness in two to four weeks with or without antimicrobial therapy. While most cases present with fever of unknown origin (FUO), previous reports have also documented atypical clinical presentation or systemic symptoms in few cases, including reports of hepatosplenic involvement. We present a case of visceral bartonellosis in an immunocompetent 15 -year-old female, who presented with a six-week history of fever and abdominal pain with hepatosplenomegaly. She recovered completely after prolonged antibiotic treatment for six weeks with doxycycline and amikacin. We emphasize that in the workup of FUO, it may be pertinent to include bartonellosis as a differential especially in cases exhibiting hepatosplenomegaly on examination along with hepatosplenic lesions on imaging.
\end{abstract}

Review began 01/29/2021 Review ended 02/01/2021 Published 02/08/2021

๑) Copyright 2021 Sharma et al. This is an open access article distributed under the terms of the Creative Commons Attribution License CC-BY 4.0., which permits unrestricted use, distribution, and reproduction in any medium, provided the original author and source are credited.
Categories: Internal Medicine, Infectious Disease

Keywords: hepatosplenic bartonellosis, visceral bartonellosis, cat scratch disease, fever of unknown origin

\section{Introduction}

Cat-scratch disease (CSD) caused by Bartonella henselae, is an infectious disease transmitted by exposure to cats, especially those infested with fleas. The disease is limited to regional lymphadenopathy but dissemination to liver, spleen, eye, bone, and central nervous system may occur. Localized disease is typically self-limiting while the disseminated disease may lead to life-threatening complications. Probable diagnoses can be made by a reported exposure to a cat, and clinical findings which can be further confirmed by serological tests.

Physicians routinely encounter fever of unknown origin (FUO). Keeping in mind, a wide differential and thorough workup are essential to come up with a definitive diagnosis and treatment. We report a case of a 15-year-old female, who presented with fever and abdominal pain of more than six-week duration, who was diagnosed with visceral bartonellosis (CSD). Imaging investigations revealed hepatosplenomegaly with multiple hepatic and splenic lesions. The patient reported recent exposure to an ill cat and $B$. henselae was suspected based on presentation and epidemiological link. She was treated with prolonged therapy of amikacin and doxycycline, which resulted in clinical as well as radiological improvement. Hepatosplenic complications of CSD are rare in immunocompetent individuals [1]. Only a few cases reported so far have presented with fever and abdominal pain [1].

\section{Case Presentation}

\section{Clinical findings}

We present the case of a 15-year-old female, with a past medical history of asthma, who presented with a six-week history of high-grade fever and left flank pain. She reported being febrile mostly every night, with temperature readings reaching up to $40^{\circ} \mathrm{C}$ as recorded at home. Her fever responded to antipyretics and was associated with malaise, fatigue, and generalized body ache. She denied any history of chills, sweating, or significant weight loss. She was noted to have dull, intermittent, left-sided non-radiating flank pain of moderate intensity with no specific aggravating or relieving factors and not associated with vomiting or any changes in bowel habits. She had recently returned from Jordan and also complained of dry cough for one month. She denied lymphadenopathy, history of sick contact, rashes, shortness of breath, joint pain, or swelling. Family history was significant for pancreatic malignancy in the father but not for any rheumatological disorder. On detailed history taking, the patient mentioned exposure to a cat one week prior to the onset of symptoms. The cat was owned by a friend and subsequently became ill to a point that the animal was treated by a veterinary doctor. The outcomes of the cat are unknown. The initial assessment revealed a temperature of $38.7^{\circ} \mathrm{C}$, pulse of 124 beats per minute, blood pressure of $119 / 73 \mathrm{mmHg}$, respiratory rate of $18 / \mathrm{min}$, and oxygen saturation of $99 \%$ on room air. Physical examination was significant 


\section{Cureus}

for hepatomegaly with liver edge palpable $2 \mathrm{~cm}$ below the coastal margin and no lymphadenopathy. The rest of the examination and review of systems were all unremarkable.

\section{Diagnostic assessment}

Initial laboratory investigations revealed microcytic anemia, high erythrocyte sedimentation rate (56 $\mathrm{mm} / \mathrm{hr}$ ), high C-reactive protein (CRP) and normal procalcitonin, mild hypercomplementemia (C3 1.96 g/L and C4 $0.44 \mathrm{~g} / \mathrm{L}$ ), and negative antinuclear antibodies (ANA), and anti-neutrophil cytoplasmic antibodies (ANCA) (Table 1).

\begin{tabular}{|c|c|c|}
\hline $\begin{array}{l}\text { Laboratory } \\
\text { investigations }\end{array}$ & Value & Lab reference \\
\hline WBC & $\begin{array}{l}4.8 \mathrm{x} \\
10^{3} / \mathrm{LL}\end{array}$ & $4.0-10 \times 10^{3} / \mathrm{uL}$ \\
\hline Hemoglobin & $9.5 \mathrm{gm} / \mathrm{dL}$ & $12-15 \mathrm{gm} / \mathrm{dL}$ \\
\hline Platelets & $\begin{array}{l}424 x \\
10^{3} / u L\end{array}$ & $150-400 \times 10^{3} / \mathrm{uL}$ \\
\hline MCV & $75.1 \mathrm{fL}$ & 83.0-101.0 fL \\
\hline ESR & $\begin{array}{l}56 \mathrm{~mm} / \mathrm{hr} \\
\text { (high) }\end{array}$ & $2-37 \mathrm{~mm} / \mathrm{hr}$ \\
\hline Haptoglobin & $\begin{array}{l}411 \\
\mathrm{mg} / \mathrm{dL}\end{array}$ & $30-200 \mathrm{mg} / \mathrm{dL}$ \\
\hline CRP & $86.5 \mathrm{mg} / \mathrm{L}$ & $0-5 \mathrm{mg} / \mathrm{L}$ \\
\hline Procalcitonin & $\begin{array}{l}0.07 \\
\mathrm{ng} / \mathrm{mL}\end{array}$ & $\begin{array}{l}<0.5 \mathrm{ng} / \mathrm{mL} \text { represents a low risk of sever sepsis and/or septic shock; }>2.0 \mathrm{ng} / \mathrm{mL} \text { represents a } \\
\text { high risk of sever sepsis and/or septic shock }\end{array}$ \\
\hline Rapid malaria test & Negative & \\
\hline ANA & Negative & \\
\hline ANCA & Negative & \\
\hline Anti-CCP Ab & Negative & \\
\hline AntI-DsDNA Ab & Negative & \\
\hline C3 & $1.96 \mathrm{gm} / \mathrm{L}$ & $0.62-1.2 \mathrm{gm} / \mathrm{L}$ \\
\hline C4 & $0.44 \mathrm{gm} / \mathrm{L}$ & $0.15-0.41 \mathrm{gm} / \mathrm{L}$ \\
\hline Quantiferon TB & Negative & \\
\hline Brucella IgG and IgM & Negative & \\
\hline Leishmania Ab & Negative & \\
\hline Cytomegalovirus IgG & Reactive & \\
\hline Cytomegalovirus IgM & $\begin{array}{l}\text { Non- } \\
\text { reactive }\end{array}$ & \\
\hline Toxoplasma Ab IgG & Reactive & \\
\hline Toxoplasma Ab IgM & $\begin{array}{l}\text { Non- } \\
\text { reactive }\end{array}$ & \\
\hline $\begin{array}{l}\text { EBV capsid antigen } \\
\text { IgG and IgM }\end{array}$ & Negative & \\
\hline $\begin{array}{l}\text { Hepatitis B surface } \\
\text { antigen }\end{array}$ & Negative & \\
\hline Hepatitis C antibody & $\begin{array}{l}\text { Non- } \\
\text { reactive }\end{array}$ & \\
\hline
\end{tabular}




\section{Cureus}

\begin{tabular}{|c|c|}
\hline HIV Ag/Ab combo & $\begin{array}{l}\text { Non- } \\
\text { reactive }\end{array}$ \\
\hline $\begin{array}{l}\text { Bartonella henselae } \\
\text { lgG }\end{array}$ & $\begin{array}{l}<1: 128 \\
\text { titer }\end{array}$ \\
\hline $\begin{array}{l}\text { Bartonella henselae } \\
\text { lgM }\end{array}$ & $<1: 20$ titer \\
\hline $\begin{array}{l}\text { Bartonella quintana } \\
\operatorname{lgG}\end{array}$ & $\begin{array}{l}<1: 128 \\
\text { titer }\end{array}$ \\
\hline $\begin{array}{l}\text { Bartonella quintana } \\
\text { lgM }\end{array}$ & $<1: 20$ titer \\
\hline
\end{tabular}

\section{TABLE 1: Laboratory investigations}

WBC, white blood cell; MCV, mean corpuscular volume; ESR, erythrocyte sedimentation rate; CRP; C-reactive protein; ANA, anti-nuclear antibodies; ANCA, anti-neutrophil cytoplasmic antibodies; CCP, cyclic citrullinated peptide; DsDNA, double-stranded DNA; IgG, immunoglobulin G; IgM, immunoglobulin M; EBV, Epstein-Barr virus; Ag/Ab, antigen/antibodies

Further investigations for tuberculosis including acid-fast bacilli smear and polymerase chain reaction (PCR) were negative from sputum and bone marrow. Culture from blood, sputum, bone marrow, and urine yielded no growth. A total of seven sets of blood culture (aerobic and anaerobic) were negative starting from the day of admission through day 5. Serum immunoglobulin G (IgG), IgM, and IgA values were within normal limits. Flow cytometry and bone marrow biopsy were negative for hematological malignancy. Kidney function, liver functions, electrolytes, lipid profile, and HbA1c were normal.

\section{Radiology}

The chest $\mathrm{x}$-ray was unremarkable. Ultrasound of the abdomen confirmed mild hepatomegaly and splenomegaly with multiple ill-defined hypoechoic lesions in the spleen. Computed tomography (CT) of the abdomen revealed hepatomegaly measuring $19.5 \mathrm{~cm}$ and splenomegaly measuring $10 \mathrm{x} 13.5 \mathrm{~cm}$ with multiple variable-size hypodense lesions with no wall enhancement (Figure 1). Results from magnetic resonance imaging (MRI) of the abdomen showed hepatosplenomegaly, multiple splenic and small illdefined hepatic lesions, and a right renal lesion (Figure 2). Whole-body positron emission tomography (PET) revealed intense hypermetabolism in multiple splenic and liver lesions (Figure 3). A transesophageal echocardiogram was negative for any masses or vegetation. Biopsy of the identifed lesions was not obtained.

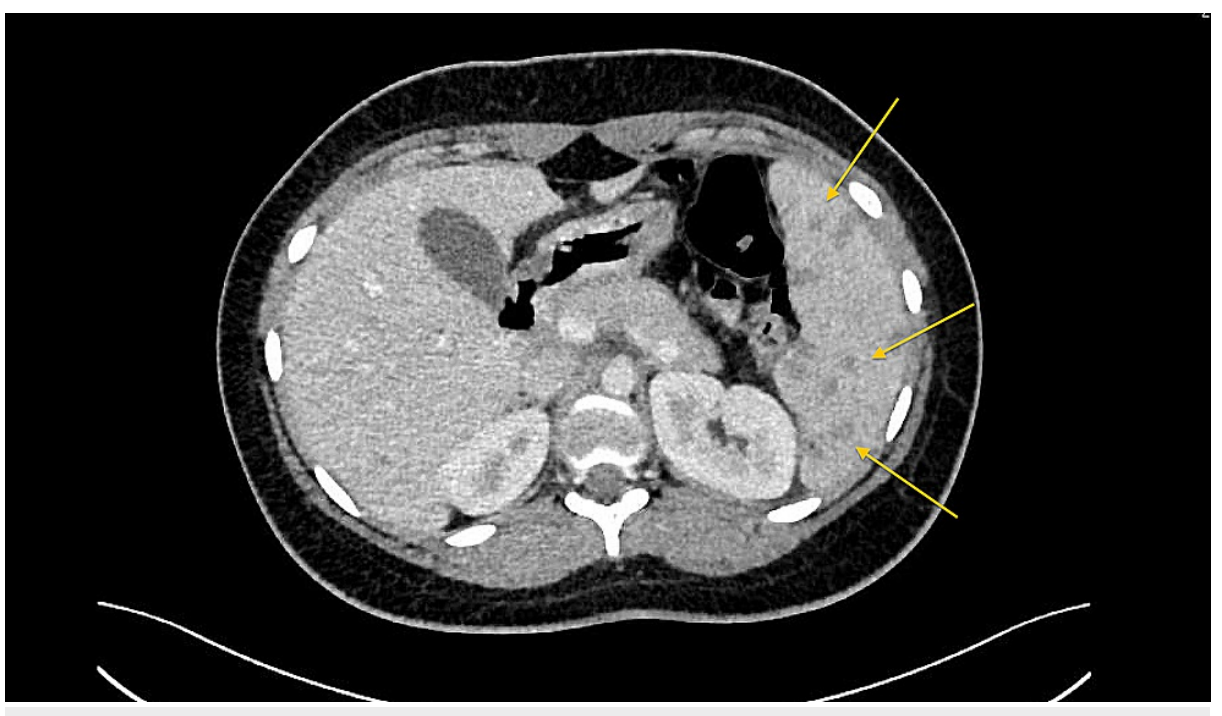

FIGURE 1: CT scan of the abdomen showing multiple hypodense lesions in the spleen (marked by yellow arrows) 


\section{Cureus}

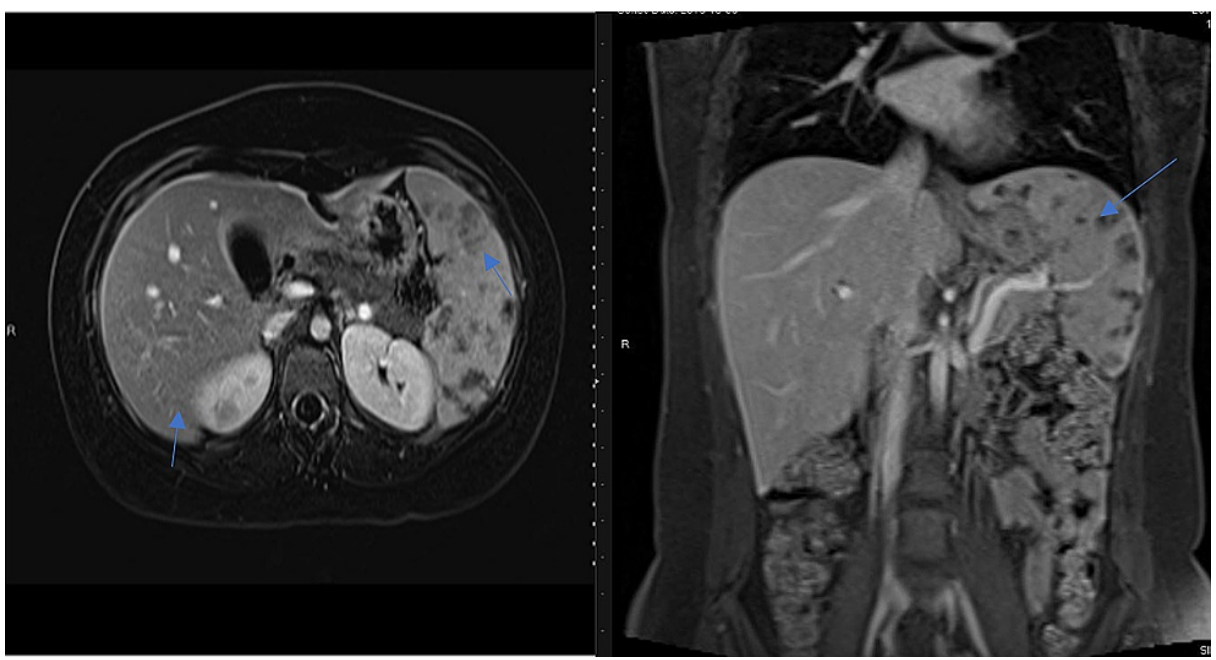

FIGURE 2: MRI of the abdomen - T1 weighted post-contrast transverse view (left image) and coronal view (right image) showing multiple hypodense lesions in the liver and spleen (marked by blue arrows)

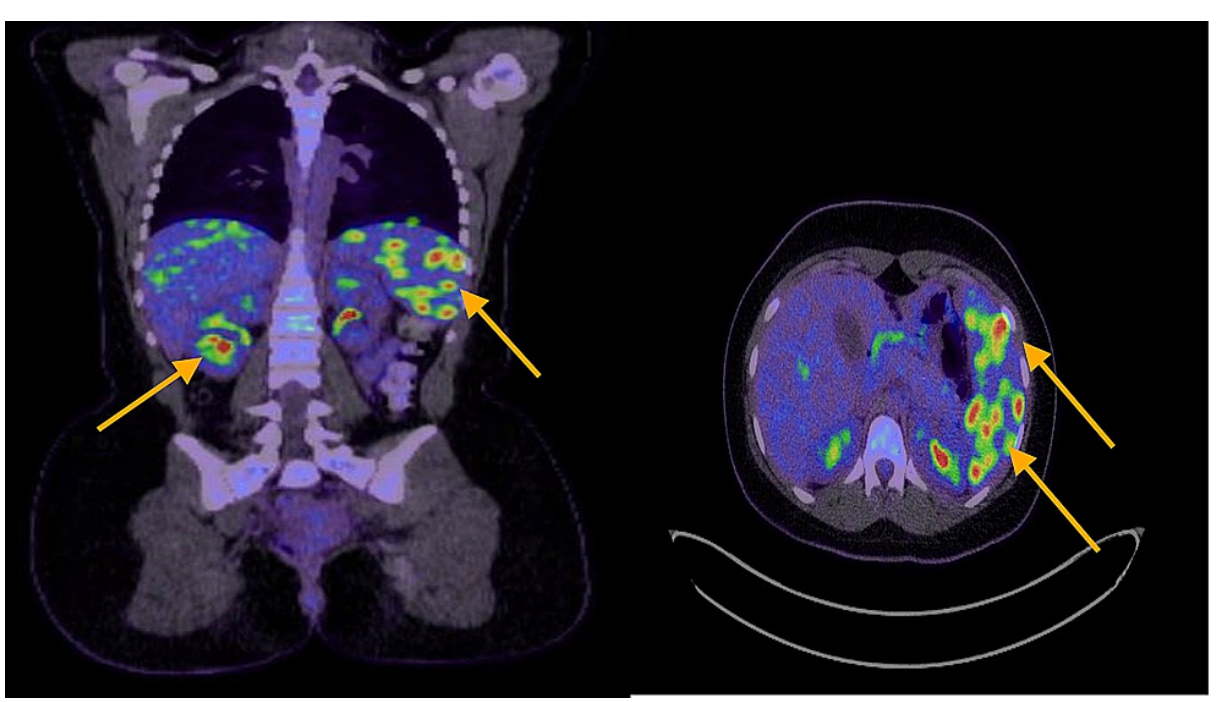

FIGURE 3: PET FDG scan showing increased multiple focal uptakes in the liver and spleen (marked by yellow arrows)

PET, positron emission tomography; FDG, fluorodeoxyglucose

\section{Therapeutic intervention}

The patient was initially started on ceftriaxone, vancomycin, and meropenem upon admission. Upon presumptive diagnosis of bartonellosis, 10 days after admission, the antimicrobial coverage was changed to amikacin for a duration of two weeks and doxycycline for six weeks.

\section{Follow-up and outcome}

After initiation of amikacin and doxycycline therapy directed against visceral bartonellosis, the patient showed significant clinical improvement in terms of fever and abdominal pain. CRP values continued to improve with treatment (Figure 4). A follow-up MRI of the abdomen two weeks after starting treatment demonstrated hepatosplenomegaly with multiple splenic lesions, which were regressing in size. The previously noted small ill-defined hepatic lesions and the right renal lesion had resolved completely. Findings were impressive for the regression of the disease, thereby supporting our diagnosis of visceral bartonellosis. 


\section{Cureus}

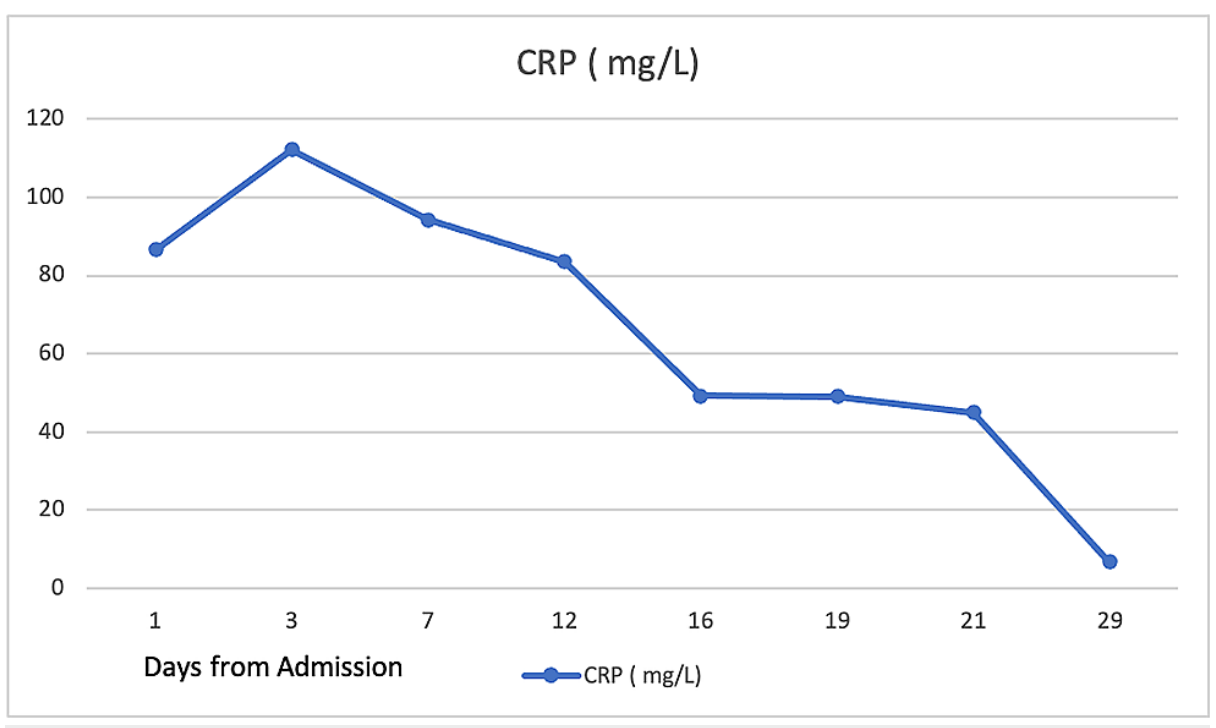

FIGURE 4: Trend of CRP with treatment against visceral bartonellosis

CRP, C-reactive protein

\section{Discussion}

CSD is an infectious disease mostly caused by $B$. henselae, a facultative intracellular Gram-negative coccobacillus, with a worldwide distribution [2]. Very rarely CSD may be caused by Afipia felis, Bartonella clarridgeiae, or unidentified fastidious organisms [3,4]. Pathogenesis of the disease remains unclear and manifestations are either local (cutaneous, regional lymphadenopathy) or systemic (ocular, visceral, neural, or musculoskeletal involvement) [5]. Cats are the primary and natural reservoir, often reported to have persistent intraerythrocytic bacteremia sometimes lasting for more than a year [6]. It is typically caused by a scratch or bites from a cat or exposure to cat fleas, usually leading to a localized cutaneous lesion or selflimiting regional lymphadenopathy [5]. Bartonella causes endothelial cell disruption, activation of a proinflammatory cascade leading to acute inflammation, subsequently followed by a rise in inflammatory markers [7]. Although transmission primarily occurs through the infected cat, however, in some cases, fleas or possibly dogs infested with fleas may also be implicated [8].

Visceral organ involvement is rare in CSD. Most of the data available are from the pediatric age group. Hepatosplenic bartonellosis, as in our case, is a rare entity in immunocompetent individuals. As far as adults are concerned, only a few cases of CSD with hepatosplenic involvement in immunocompetent individuals have been reported [1]. When relevant, CSD must be considered in the differential diagnosis of FUO. Probable diagnosis can be made by history and presentation of typical features. Serological testing such as enzyme immunoassay or indirect fluorescence assay is useful in confirming the diagnosis $[9,10]$. A negative serological test cannot rule out CSD in highly suspected cases. The recommendation is to do a paired serological test, which was unfortunately not done in our case due to the lack of availability. Lymph node or tissue biopsy may be performed in certain circumstances to confirm the diagnosis or when alternative diagnoses such as tuberculosis or lymphoma are suspected. A biopsy may reveal necrotizing granulomatous inflammation, and Warthin-Starry stain may demonstrate pleomorphic B. henselae bacilli [5]. Imaging techniques are needed to diagnose visceral involvement in CSD. Ultrasound, CT, and MRI may be useful for the assessment of hepatic, splenic, or renal involvement. In our case, we performed an ultrasound, CT, MRI as well as a PET scan to assess the degree of visceral involvement. Imaging may also be helpful to follow up and evaluate the clinical response to treatment. We appreciated a significant radiological resolution of intra-abdominal lesions in our case. Treatment of hepatosplenic CSD and FUO is with azithromycin plus rifampin; alternatively, rifampin and gentamycin can be used [11-13]. High-dose azithromycin (500 mg PO/IV for five days) alone could be used for immunocompetent adults [1]. Our case was treated with two weeks of amikacin and six weeks of doxycycline to potentially cover Chlamydia psittaci or other atypical infections although visceral bartonellosis was most likely the cause based on presentation, epidemiological link, and response to treatment.

Currently, no specific markers or titers are available for monitoring patient's responses to treatment. We monitored the response to treatment with CRP and follow-up abdominal imagining. Although further imaging may not be required, follow-up imaging at six months after the completion of treatment may be warranted to document regression or resolution of lesions.

\section{Conclusions}


CSD caused by Bartonella henselae is an important differential while evaluating for fever of unknown origin. Hepatosplenic complications may occur, although rare in immunocompetent individuals. Advanced imaging, and Bartonella serology coupled with detailed history maybe useful in diagnosis of such cases.

\section{Additional Information \\ Disclosures}

Human subjects: Consent was obtained or waived by all participants in this study. Conflicts of interest: In compliance with the ICMJE uniform disclosure form, all authors declare the following: Payment/services info: All authors have declared that no financial support was received from any organization for the submitted work. Financial relationships: All authors have declared that they have no financial relationships at present or within the previous three years with any organizations that might have an interest in the submitted work. Other relationships: All authors have declared that there are no other relationships or activities that could appear to have influenced the submitted work.

\section{References}

1. García JC, Núñez MJ, Castro B, Fernández JM, Portillo A, Oteo JA: Hepatosplenic cat scratch disease in immunocompetent adults. Medicine (Baltimore). 2014, 93:267-279. 10.1097/md.0000000000000089

2. Szelc-Kelly CM, Goral S, Perez-Perez GI, Perkins BA, Regnery RL, Edwards KM: Serologic responses to Bartonella and Afipia antigens in patients with cat scratch disease. Pediatrics. 1995, 96:1137-1142.

3. Giladi M, Avidor B, Kletter Y, et al.: Cat scratch disease: the rare role of Afipia felis . J Clin Microbiol. 1998, 36:2499-2502. 10.1128/JCM.36.9.2499-2502.1998

4. D L Kordick, E J Hilyard, T L Hadfield, Wilson KH, Steigerwalt AG, Brenner DJ, Breitschwerdt EB: Bartonella clarridgeiae, a newly recognized zoonotic pathogen causing inoculation papules, fever, and lymphadenopathy (cat scratch disease). J Clin Microbiol. 1997, 35:1813-1818. 10.1128/JCM.35.7.18131818.1997

5. Bass JW, Vincent JM, Person DA: The expanding spectrum of Bartonella infections: II. Cat-scratch disease . Pediatr Infect Dis J. 1997, 16:163-179. 10.1097/00006454-199702000-00002

6. Jacomo V, Kelly PJ, Raoult D: Natural history of Bartonella infections (an exception to Koch's postulate) . Clin Diagn Lab Immunol. 2002, 9:8-18. 10.1128/cdli.9.1.8-18.2002

7. Dehio C: Molecular and cellular basis of Bartonella pathogenesis . Annu Rev Microbiol. 2004, 58:365-390. 10.1146/annurev.micro.58.030603.123700

8. Zangwill KM, Hamilton DH, Perkins BA, et al.: Cat scratch disease in Connecticut. Epidemiology, risk factors, and evaluation of a new diagnostic test. N Engl J Med. 1993, 329:8-13. 10.1056/NEJM199307013290102

9. Florin TA, Zaoutis TE, Zaoutis LB: Beyond cat scratch disease: widening spectrum of Bartonella henselae infection. Pediatrics. 2008, 121 :e1413-e1425. 10.1542/peds.2007-1897

10. Margileth AM: Recent advances in diagnosis and treatment of cat scratch disease . Curr Infect Dis Rep. 2000, 2:141-146. 10.1007/s11908-000-0026-8

11. Margileth AM: Antibiotic therapy for cat-scratch disease: clinical study of therapeutic outcome in 268 patients and a review of the literature. Pediatr Infect Dis J. 1992, 11:474-478.

12. Collio PJ: Cat-scratch disease: therapy with trimethoprim-sulfamethoxazole. Am J Dis Child. 1992, 146:397399.

13. Shorbatli LA, Koranyi KI, Nahata MC: Effectiveness of antibiotic therapy in pediatric patients with cat scratch disease. Int J Clin Pharm. 2018, 40:1458-1461. 10.1007/s11096-018-0746-1 